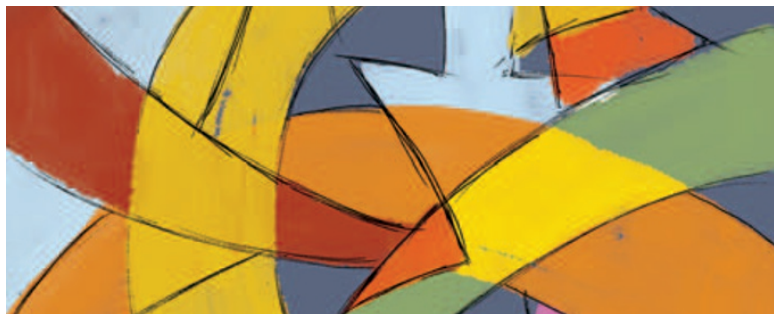

DISEASE GENETICS

\section{A pathway to complexity}

Single genes that predispose to complex diseases usually only have a small effect on disease risk and are therefore poor predictive markers of disease. Instead, the combined action of multiple genes within a pathway might tell us more about susceptibility to complex diseases. Recent work by Maraganore and colleagues shows that focusing on polymorphisms in genes that are involved in the axonguidance pathway yields strong predictors of risks that are associated with Parkinson disease (PD).

The axon-guidance pathway, which wires the brain during fetal development by driving axons towards their synaptic targets, has been implicated as a candidate for the development of PD. The authors had previously identified a SNP in a gene involved in this pathway that was associated with PD susceptibility. So they decided to assess whether variation in multiple genes that function in this pathway has a bigger effect on predisposing to PD compared with a single-gene variation.

They used data from a previous whole-genome association study of SNPs in 443 PD cases and 443 controls from unaffected siblings. They identified 128 genes that were involved in the axon-guidance pathway, for which 1,460 SNPs had been genotyped. Using a bioinformatic approach they constructed models that sorted subsets of SNPs into groups that were predictive of PD susceptibility, of survival with no PD and of age at PD onset. Compared with models constructed from random selected SNPs, the axon-guidance pathway derived models that are very robust: they could predict 90 -fold differences in PD susceptibility and 20-year differences in age at disease onset, indicating that gene variation within this pathway has a large effect on predisposition. Remarkably, predictive models that were constructed using the same pathway and genes but SNPs from a different whole-genome association study yielded similar results. Analysis of a geneexpression profiling dataset for PD showed that axon-guidance pathway genes were differentially expressed in the brains of PD-affected individuals compared with the controls, further supporting the relevance of this pathway in PD.

Beyond its important implications for PD, this study demonstrates that shifting from single-gene to pathway approaches when studying complex diseases enhances the disease-predictive power of association studies.

Francesca Pentimalli

ORIGINAL RESEARCH PAPER Lesnick, T. G. et al. A genomic pathway approach to a complex disease: axon guidance and Parkinson disease. PLoS Genet. 3, e98 (2007) FURTHER READING Balding, D. J. A tutorial on statistical methods for population association studies. Nature Rev. Genet. 7, 781-791 (2006)

\title{
Introducing the mirtron
}

Introns are a common source of canonical microRNAs, but new research shows that they can also encode a separate class of miRNA precursors - mirtrons

- that are processed by a distinct, Drosha-independent pathway.

Animal microRNAs are processed in two stages: first, a hairpin (pre-miRNA) is cleaved from a longer transcript (pri-miRNA) by Drosha; second, this hairpin is exported to the cytoplasm and cleaved into two by Dicer to produce the functional microRNA. The Bartel and Lai laboratories now describe how Drosophila melanogaster introns can be processed initially by the intron pathway before being cleaved by Dicer into functional microRNAs.

Bartel and colleagues identified $14 \mathrm{D}$. melanogaster introns with the sequence and predicted secondary structure of pre-miRNAs but none of the extended structure and sequence of pri-miRNAs. Lai and colleagues detected expression of the processed versions of these introns in various tissues and developmental stages, and both groups then demonstrated that the products of mirtrons could repress reporter constructs.

To demonstrate that mirtrons depend on splicing and not Drosha, both groups looked at cis and trans factors. First, they showed that mutation of the nucleotides that are required for splicing abolished production of the mirtrons. Second, they knocked down expression of the lariat debranching enzyme, which resolves spliced introns into a form that can then form hairpins, and found reduced amounts of mirtrons but not of canonical miRNAs.

\section{DEVELOPMENT}

\section{PGCs' epigenetic travelogue}

Unlike the worm and the fly, mammals set their germ cells apart rather late during embryonic development. Once selected from among their somatic neighbours, germ cells must reprogramme their genome and journey to the part of the embryo where the future gonad will be. A recent report reveals for the first time the dynamics of this reprogramming and the associated cellular events during their journey.

To monitor epigenetic changes, transcriptional activity and cellcycle stages during primordial germ cell (PGC) migration, the authors mainly relied on immunohistochemistry, carried out in mice in which these cells are genetically marked with EGFP. Using antibodies against specific histone modifications, Seki et al. showed that as migration begins, around embryonic day E7.5, the levels of di- and monomethylated histone $\mathrm{H} 3$ lysine 9 ( $\mathrm{H} 3 \mathrm{~K} 9 \mathrm{me} 2$ and $\mathrm{H} 3 \mathrm{~K} 9 \mathrm{me} 1$, respectively) - repressive marks - begin to drop and continue to do so until E8.75. But trimethylated histone H3 lysine 27 (H2K27me3) - another repressive mark - appeared elevated only at E8.25 and increased over time.

Given this erasure of $\mathrm{H} 3 \mathrm{~K} 9 \mathrm{me} 2$ and $\mathrm{H} 3 \mathrm{~K} 9 \mathrm{me} 1$, Seki et al. looked for a phosphorylated form of RNA polymerase II, which is associated with active transcription. Contrary to expectations, they found that its levels in PGCs were low at E8.75, suggesting that transcription is blocked in these cells. Using another antibody to detect the RNA polymerase II form that is associated with transition from transcriptional initiation to promoter clearance, the authors show that it is at this stage that transcription is blocked in PGCs. From these and other results, the 
Conversely, knocking down Drosha affected canonical miRNAs but not mirtrons.

In contrast to these differences at the first stage of processing, mirtrons and miRNAs depend on similar trans factors at the second stage of processing. Lai and colleagues showed that both depend on Exportin 5 for transfer to the cytoplasm and Argonaute 1 in order to regulate mRNAs, and both groups showed that mirtrons require Dicer for processing the pre-miRNAs into mature products.

The prevalence of mirtrons in other species remains to be seen - it might be that they are more common in species such as flies and nematodes, in which average intron length is close to that of a pre-miRNA, than they are in mammals. Indeed, the Bartel laboratory identified several mirtrons in Caenorhabditis elegans. It will also be interesting to better understand their evolutionary dynamics - a proportion of introns might be processed as mirtrons simply because the processing machinery is there If so, mirtrons would be lost rapidly through selection if they were deleterious, or slowly through genetic drift if they were neutral, and would be retained only if they were beneficial. An important next step will be to catalogue such random or accidental mirtrons as well as the putatively functional ones that have been classified so far.

Patrick Goymer

ORIGINAL RESEARCH PAPERS Ruby, J. G., Jan, C. H. \& Bartel, D. P. Intronic microRNA precursors that bypass Drosha processing. Nature 24 June 2007 (doi:10.1038/nature05983) | Okamura, K. et al. The mirtron pathway generates microRNA-class regulatory RNAs in Drosophila. Cell 28 June 2007 (doi:10.1016/j.cell.2007.06.028) FURTHER READING Chen, K. \& Rajewsky, N. The evolution of gene regulation by transcription factors and microRNAs. Nature Rev. Genet. 8 . 93-103 (2007) authors propose that during migration PGCs become arrested at $\mathrm{G} 2$ phase of the cell cycle, their $\mathrm{H} 3 \mathrm{~K} 9$ me 2 levels drop and transcription is blocked (in a chromatin-independent manner); subsequently, H3K27me3 levels increase and transcription resumes approximately when PGCs are also released from the $\mathrm{G} 2$ arrest.

Delving into upstream mechanisms, Seki et al. showed that, unlike in the fly and the worm, nanos is not required for epigenetic reprogramming and transcriptional suppression in PGCs. Jumonji C (JmjC)-domain-containing histone demethylases are expressed in PGCs and their somatic neighbours alike, leading the authors to reason that PGCs might possess additional mechanism(s) to direct genomewide $\mathrm{H} 3 \mathrm{~K} 9 \mathrm{me} 2 / \mathrm{me} 1$ loss. As GLP (also known as EHMT1) - a histone methyltransferase - and its partner G9a (also known as EHMT2) are downregulated around E7.5 and E9.0, respectively, in PGCs, the authors propose that they are responsible for $\mathrm{H} 3 \mathrm{~K} 9 \mathrm{me} 2 / \mathrm{me} 1$ loss in these cells.
This elegant work provides an important insight into migrating PGCs. Further work will be needed to link these early events with the subsequent events that take place in the genital ridges - the location of the future gonad.

Magdalena Skipper

ORIGINAL RESEARCH PAPER Seki, Y. et al.

Cellular dynamics associated with the genomewide epigenetic reprogramming in migrating primordial germ cells in mice. Development $\mathbf{1 3 4}$, 2627-2638 (2007)

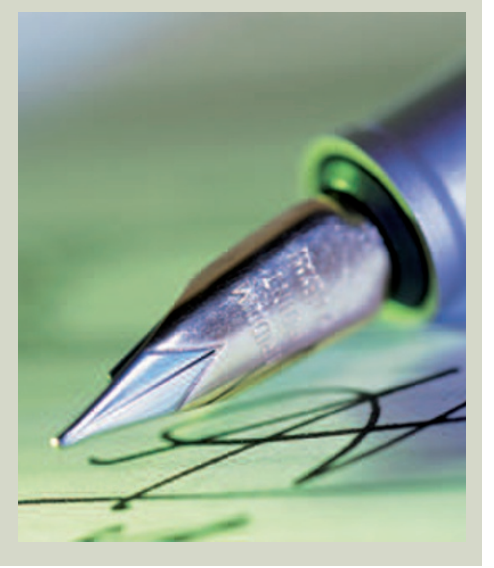

\section{IN BRIEF}

\section{$\Rightarrow$ GENETIC SCREENS}

A genome-wide transgenic RNAi library for conditional gene inactivation in Drosophila.

Dietzl, G. et al. Nature 12 July 2007 (doi: 10.1038/nature05954)

This paper reports the generation and validation of a large-scale transgenic library for RNAi screening in Drosophila melanogaster, allowing gene functions to be analysed in all tissues and developmental stages. The transgenes contain inverted-repeat constructs, and expression is driven using the GAL4-UAS system. The authors generated 22,270 transgenic lines, which cover $88 \%$ of the D. melanogaster genes that are predicted to encode proteins. Most of the transgenes in these lines were confirmed to be functional in molecular and phenotypic assays.

\section{$\Rightarrow$ BEHAVIOURAL GENETICS}

Natural polymorphism affecting learning and memory in Drosophila.

Mery, F. et al. Proc. Natl Acad. Sci. USA 12 July 2007 (doi:10.1073/ pnas.0702923104)

Previously, the only naturally occurring polymorphisms with possible influences on learning and memory have been identified in humans, making them difficult to study. This paper shows that an allelic variant at the Drosophila melanogaster foraging locus confers improved short-term memory, but poorer long-term memory, whereas the reverse is true for a second allele of this gene. The authors suggest that this polymorphism might be an evolutionary trade-off between the two forms of memory. This finding opens up the possibility of studying the evolution of learning and memory in a model organism.

\section{$\Rightarrow$ EVOLUTION}

Selection for robustness in mutagenized RNA viruses.

Sanjuán, R. et al. PLoS Genet. 3, e93 (2007)

Selection should favour fast-replicating viral populations over slower ones - that is the definition of fitness in such populations. However, can the slower replicators be favoured if they are more robust to the effects of deleterious mutations? The authors competed two populations of vesicular stomatitis virus, both of which resided at local peaks on the adaptive landscape, but one of which was on a shorter but also shallower and therefore more robust peak. Above a certain threshold concentration of mutagens in the environment, the slower, more robust population outcompeted the faster replicators.

\section{$\Rightarrow$ RNA WORLD}

A mammalian microRNA expression atlas based on small RNA library sequencing.

Landgraf, P. et al. Cell 129, 1401-1414 (2007)

In this resource paper, the authors report the sequencing of more than 250 small-RNA libraries from 26 organs and cell types, including malignant haematopoietic cells, in humans and rodents. For a subset of these RNAs, they determined genomic organization, conservation patterns and expression profiles. Interestingly, they report that only a small subset of microRNAs (miRNAs) accounts for the differences in miRNA expression profiles between cell types and tissues. The newly developed tools for miRNA-profile analysis and presentation should facilitate future research and contribute to the growth of this miRNA gene atlas. 